\title{
Was Naturheilkunde bei unerfülltem Kinderwunsch leisten kann
}

Autorin: Ingrid Gerhard

\section{ZUSAMMENFASSUNG}

Die naturheilkundliche Behandlung einer Sterilität birgt große Chancen. Verschiedene Studien zeigen, dass die Verbesserung der Lebensstilfaktoren die spontane Schwangerschaftsrate mindestens verdoppeln kann. Die zuverlässigsten Daten liegen zur Optimierung des Lebensstils, der Ernährung und Nahrungsergänzung vor. Nach der Basisdiagnostik zu den Sterilitätsursachen können jedoch auch weitere naturheilkundliche Methoden ergänzend genutzt werden, um den unerfüllten Kinderwunsch zu behandeln.

\section{Schlüsselwörter}

Sterilität, unerfüllter Kinderwunsch, Infertilität, Kinderwunschbehandlung, In-vitro-Fertilisation

\section{ABSTRACT}

The naturopathic treatment of sterility offers great opportunities. Various studies show that the improvement of lifestyle factors can at least double the spontaneous pregnancy rate. The most reliable data is available for the optimization of lifestyle, nutrition and nutritional supplementation. After the basic diagnosis of the causes of sterility, other naturopathic methods can also be used to treat the unfulfilled desire to have children.

Keywords

Sterility, unfulfilled desire to have children, infertility, fertility treatment, in vitro fertilization.

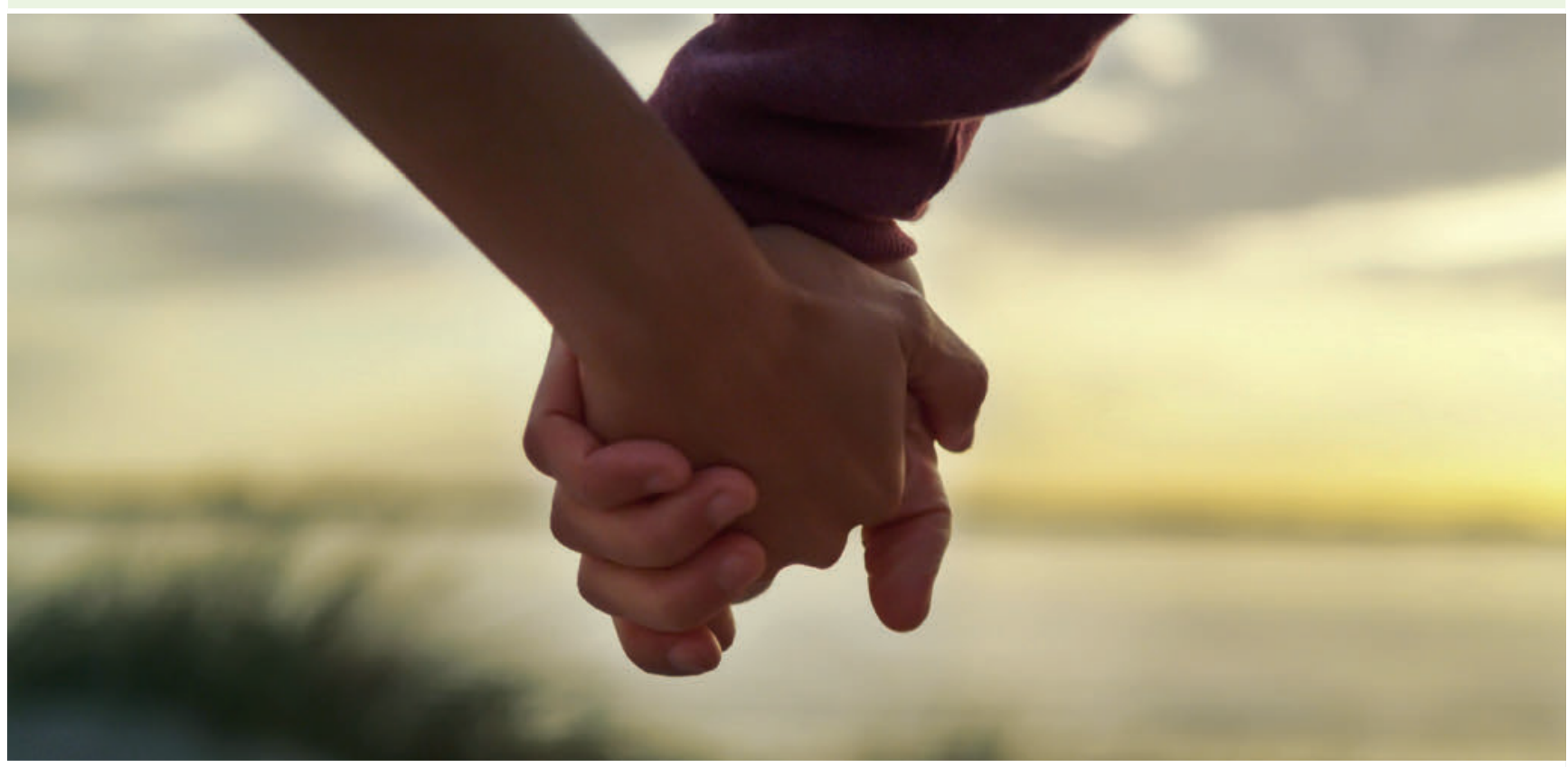

Abb. 1 Ein unerfüllter Kinderwunsch ist mit einem hohen psychischen Stresslevel verbunden. Zeit für Gespräche und das Erleben positiver Momente sind daher besonders wichtig. @ icsnaps/Adobe Stock

Seit einigen Jahren wird das Problem des unerfüllten Kinderwunsches immer drängender. Das hat verschiedene Gründe:

- Kontinuierlich steigt das Alter der Frauen mit Wunsch nach einem ersten Kind an. Während noch in den Sechzigerjahren das durchschnittliche Alter bei der ersten Schwangerschaft unter 25 Jahren lag, liegt es inzwischen deutlich über 30 Jahren. Die Ansprüche an das Leben, die veränderten Familienstrukturen sowie die Berufstätigkeit von Frauen haben dazu geführt, dass das Kinderkriegen gerne hinausgeschoben wird. Das ändert aber nichts daran, dass die fertile Phase der Frau zeitlich begrenzt ist und die Qualität der Eizellen mit zunehmendem Alter schlechter wird.

- Schädliche Umweltbelastungen haben zugenommen. Täglich werden neue Chemikalien in Umlauf gebracht, deren Wirkungen auf die Fortpflanzung und den weiblichen Organismus nie geprüft wurden. Erst 
Jahrzehnte später werden nach und nach die Folgen klar: Pestizide, Schwermetalle, Phthalate, Flammschutzmittel, Kosmetika usw. haben neben ihren toxischen Wirkungen, bspw. auf die Mitochondrien, oft auch Wirkungen auf das Hormongleichgewicht (Endokrine Disruptoren, Xenoöstrogene).

- Durch die geänderten Ernährungsgewohnheiten (Fast Food, Kantine, Fertigkost, Verwendung von Konservierungsstoffen, einfacher Kohlenhydrate und viel tierischem Protein, wenig Pflanzenkost) und die oft niedrigwertige Industrienahrung verarmt der Organismus an Vitalstoffen und Antioxidantien, sodass er die Umweltbelastungen nicht abfangen kann.

- Hinzu kommen Bewegungsmangel, Stress am Arbeitsplatz und in der Freizeit, zu seltener Aufenthalt in der Natur, Elektrosmog.

- Die anfänglich hochgespannten Erwartungen, die in die reproduktionsmedizinischen Techniken gesetzt wurden, konnten sich nur für einen Teil der Kinderwunschpatienten erfüllen. Wiederholte Versuche, hohe Eigenkosten, Hormonkuren und Erfolgsdruck haben viele Paare an ihre Grenzen sowohl als Individuum als auch als Paar gebracht.

Aus dieser Aufzählung wird deutlich, dass das Vorgehen bei unerfülltem Kinderwunsch nicht schematisch, sondern individuell erfolgen muss. Hier sehe ich große Chancen für die Naturheilkunde. Denn der naturheilkundlich orientierte Arzt ist es gewohnt, nicht nur ein Organ zu behandeln, sondern komplexe Zusammenhänge zu erkennen und individuell den Patienten und nicht nur die Diagnose Kinderwunsch zu behandeln.

Bereits beim Erstkontakt mit der Patientin können durch eine eingehende Anamnese Hinweise auf die Sterilitätsursachen gefunden werden. Ehe mit einer naturheilkundlichen Behandlung begonnen wird, sollte gesichert sein, dass die Basis-Sterilitätsdiagnostik erfolgt ist: Basaltemperaturmessung, Spermiogramm des Partners, Tubenfunktion, eventuell Sims-Huhner-Test und Hormonbestimmungen. Auch bei biphasischem Zyklus sollten die Schilddrüsenwerte und SD-Antikörper bestimmt werden.

Bei der Behandlung ist im Einzelnen wie folgt vorzugehen.

\section{Lifestyle optimieren}

\section{Umweltsanierung}

Dem infertilen Paar sollte deutlich erklärt werden, dass „in schlechter Erde kein guter Salat wachsen kann“. Das bedeutet, dass wir heute wissen, dass epigenetische Faktoren über mehrere Generationen die Gesundheit der Nachkommen beeinflussen. Mit diesem Verständnis wird die Bereitschaft eines Pares vielleicht größer sein, folgende Umweltbedingungen zu schaffen:

- Mit dem Rauchen aufhören.
- Umweltverträgliche und schadstoffarme Produkte in Haushalt, Wohnung und am Arbeitsplatz benutzen.

- Kosmetische Produkte und Hygieneartikel gezielt ohne hormonaktive Substanzen auswählen (hierbei hilft die App Toxfox vom Bund für Umwelt und Naturschutz Deutschland e. V. und die App CodeCheck).

- Schadstoffarme Kleidung aus Naturmaterialen bevorzugen.

- Wasserfilter für Trink- und Kochwasser anschaffen, da unsere Wasserwerke Medikamente, hormonaktive Substanzen und Pestizide aus dem Wasser nicht herausfiltern können. Wasser aus Plastikflaschen meiden, da Plastikpartikel immer mitgetrunken werden.

- Alle Elektrogeräte aus dem Schlafzimmer verbannen, eventuell Netzfreischalter einbauen. Achtsamer Umgang mit Computer, Handy, Laptop, z. B. Handy nicht in Hosentasche stecken, Laptop nicht auf den Schoß stellen, sondern Abstand zur Strahlenquelle halten.

\section{Innenweltsanierung}

Toxische Belastungen können im Sinne von chronischen Entzündungen, Silent Inflammation und Störfeldern sowohl beim Mann verantwortlich sein für zum Teil hochpathologische Spermiogramme als auch bei der Frau zur Sterilität und Infertilität führen. Naturheilkundeärzten ist das seit langer Zeit bekannt.

Von Reproduktionsmedizinern unterschätzt ist die Problematik des gestörten Darms und - sehr aktuell - der Parodontitis [5][12]. Mit der aMMP-8-Biomarker-Diagnostik (Periosafe: www.periopreventioncenter.de/) steht jedem Arzt ein einfaches Präventionssystem in der eigenen Praxis zur Verfügung. Die Therapieansätze mit Zahnreinigung, orthomolekularer Therapie und Mikrobiota haben sich in der Praxis bereits als sehr erfolgreich erwiesen, um bei männlicher und weiblicher Infertilität (inklusive Endometriose) Gesundheit und Schwangerschaften auf natürlichem Wege zu ermöglichen [25].

\section{Ernährungsoptimierung}

Untergewichtige Frauen sollten zur Gewichtszunahme motiviert und darin unterstützt werden. Denn nur bei einem optimalen Fettanteil kann mit guten Ovulationen und einer ungestörten Schwangerschaft gerechnet werden. Übergewichtige und adipöse Frauen müssen keine CrashDiät machen, um rasch schwanger zu werden. Meine Erfahrungen zeigen, dass durch eine optimierte Ernährung, die automatisch zu einer verbesserten Stoffwechselfunktion und einem langsamen Gewichtsverlust führt, die Prognose auf einen spontanen Schwangerschaftseintritt deutlich erhöht wird. Dazu kann schon eine durchschnittliche Gewichtsabnahme von $5 \%$ reichen.

Übergewichtige und adipöse Männer haben ein schlechteres Spermiogramm als normalgewichtige Männer. Besonders wenn bereits ein metabolisches Syndrom vorliegt, ist die Spermienqualität deutlich eingeschränkt. Allein durch 


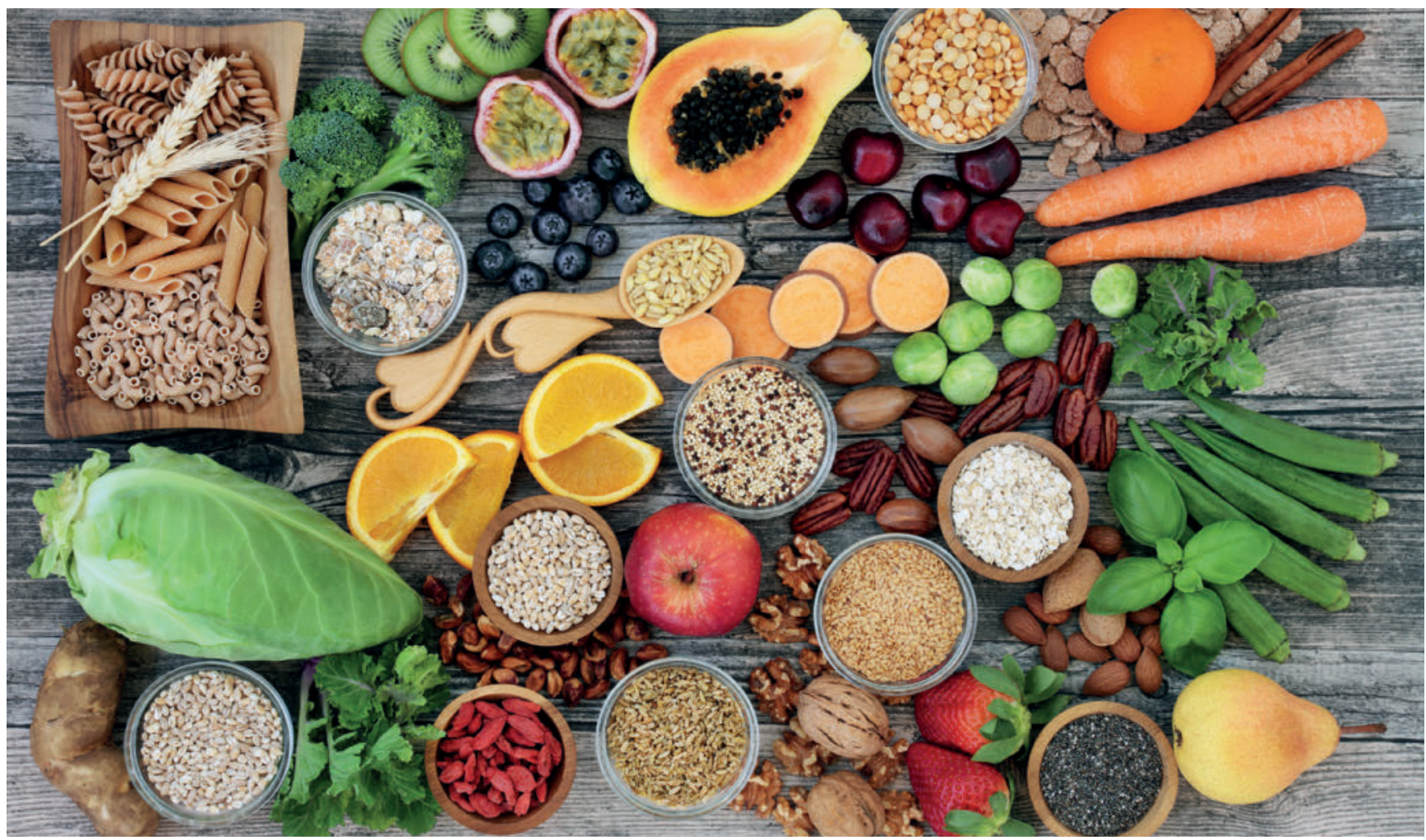

Abb. 2 Die Ernährung sollte mindestens drei Monate vor der erwünschten Konzeption auf eine gesunde, nährstoffreiche Kost umgestellt werden. (๑) marilyn barbone/Adobe Stock

Veränderungen des Lifestyles und Verbesserung der Ernährung lässt sich die Anzahl mobiler Spermien erhöhen.

Die Ernährung sollte pflanzenbetont sein, mit einem hohen Anteil an komplexen Kohlenhydraten (Vollkorngetreide, Hülsenfrüchte, Gemüse, Obst), bevorzugt aus regionalem Anbau und biozertifiziert. Es gibt inzwischen einige Studien, die einen Zusammenhang zwischen dem Verzehr von Isoflavonoiden oder Lignanen mit verbesserten Fertilitätsparametern zeigten. Die Höhe der Lignanausscheidung im Urin korrelierte bspw. positiv mit einer kürzeren Dauer bis zur Konzeption [20]. Mit zunehmendem Verzehr von Sojaisoflavonen erhöhte sich bei In-vitro-Fertilisation (IVF) die Lebendgeburtenrate [24].

Fast Food, Softgetränke und Nahrungsmittel mit einfachen Kohlenhydraten (Zucker, Mehle, Alkohol) sollen gemieden werden. Fleisch, ebenfalls biozertifiziert, sollte nur 1-2 $\times$ pro Woche und Fisch höchstens $1 \times$ pro Woche gegessen werden. Aus ökologischen Gesichtspunkten und in Anbetracht der starken Belastung mit Umweltgiften kann auf Fisch verzichtet werden, allerdings sollten dann die wichtigen Omega-3-Fettsäuren als Nahrungsergänzungen eingenommen werden. Der Verzehr von Kuhmilchprodukten sollte reduziert werden, da die Intensiv-Viehwirtschaft zu erhöhten Hormonkonzentrationen und anderen unerwünschten Stoffen in der Milch geführt hat. Es kann teilweise auf Ziegen- und Schafsmilchprodukte ausgewichen werden.
Besonders strenge Ernährungsempfehlungen gelten für Frauen mit Myomen, Endometriose oder polyzystischen Ovarien (PCO). Nahrungsmittel, die viele endokrine Disruptoren (Milchprodukte) und viele entzündungsfördernde Substanzen (Fleisch, Milch) enthalten oder den Zuckerstoffwechsel belasten (Einfachzucker), sind zu reduzieren.

\section{Stressregulation}

Gespräche mit dem Paar über die Hintergründe des Kinderwunsches, belastende Lebensfaktoren, Partnerschaft und Lebensentwürfe können sehr wichtig sein. Dafür braucht es nicht unbedingt eine Psychotherapie. Bei einem psychologischen Projekt zum unerfüllten Kinderwunsch, das vor einigen Jahren multizentrisch in Deutschland durchgeführt wurde, ergab sich in meiner Arbeitsgruppe, dass sich durch eine dreimalige Paarberatung zwar nicht kurzfristig die spontane Schwangerschaftsrate erhöhte. Die Paare gaben jedoch übereinstimmend an, dass sie sich dadurch deutlich entlastet gefühlt hätten, das Verhältnis zum Partner sich gebessert hätte und sie optimistisch und realistisch der weiteren Kinderwunschbehandlung entgegensehen konnten.

Die Mehrzahl der Kinderwunschpaare hat heute Bewegungsmangel. Sport sollte nicht zum Freizeitstress werden, es ist den Paaren aber zu empfehlen, $3 \times$ pro Woche mindestens eine Stunde Sport zu machen (bevorzugt im Freien), um den Stoffwechsel zu aktivieren und die Sauerstoffversorgung zu verbessern. „Dauersitzer“ sollten re- 
gelmäßig aufstehen und laufen, um die Durchblutung der Beckenorgane zu verbessern. Bei pathologischen Spermiogrammen kann allein diese Maßnahme „Wunder wirken“.

Entspannungsmethoden und Visualisierungen können für Frauen sehr wichtig sein. Da bei Stress zu viel Progesteron zur Produktion von Nebennierenhormonen verbraucht wird, leidet heute die Mehrzahl der Frauen an einer Lutealinsuffizienz. Methoden wie Yoga, Qigong, Tai-Chi oder Achtsamkeitstraining können dazu beitragen, dass das Stressniveau sinkt und sich der Zyklus verbessert.

Viele Frauen haben den Bezug zu ihrem Schoßraum, zu ihrer Gebärmutter, verloren. Hier setzen verschiedene Methoden an, die von Therapeutinnen angeboten werden: Gespräche mit der Gebärmutter, Fruchtbarkeitsmassagen, Rituale zur Einladung der Seele eines Kindes. Manchem Therapeut werden diese Methoden esoterisch erscheinen, sie bieten Frauen, die unter den technischen Prozeduren der Reproduktionsmedizin am meisten zu leiden haben, jedoch eine Hilfe, das Vertrauen in den eigenen Körper und die eigene Kraft wiederzuerlangen.

\section{Therapeutische Ansätze}

\section{Entgiftung}

Ehe mit einer gezielten Therapie zur verbesserten Eireifung oder Verbesserung des Spermiogramms begonnen wird, ist es sinnvoll, eine Entgiftungsbehandlung durchzuführen. Diese hat zum Ziel, nicht nur sog. Umweltgifte zur Ausscheidung zu bringen, sondern auch die Funktion der Entgiftungsorgane zu verbessern, da diese gerade bei Frauen oft durch langjährige Hormoneinnahme oder Medikamente gestört ist. Eine Entgiftungsbehandlung könnte eine Fasten- oder Kräuterteekur, eine unterstützende Therapie für Leber, Nieren und Darm oder die gezielte Gabe von Chelatbildnern sein. In der Entgiftungsphase, die für beide Partner sinnvoll ist, sollte nach Möglichkeit noch keine Schwangerschaft eintreten. Naturheilkundlich arbeitende Kollegen berichten immer wieder, dass allein diese Maßnahmen ausreichen, um deutlich verbesserte Spermiogramme zu erhalten und den Eintritt spontaner Schwangerschaften zu ermöglichen.

\section{Nahrungsergänzungen}

In den vergangenen Jahren erschien eine regelrechte Flut von Publikationen, die sich mit der orthomolekularen Therapie und der Verbesserung der männlichen Fertilität befassen. Sowohl in vitro als auch in Tierversuchen und beim Menschen wurden die unterschiedlichsten Produkte eingesetzt, meist einzeln, seltener in Kombination, sodass die Beurteilung der Effektivität sehr schwierig ist. Inzwischen ist es dank der In-vitro-Fertilisierung möglich, auch bei Frauen in kurzer Zeit den Effekt einer Nahrungsergänzung auf die Fertilisierungsrate der Eizellen und die frühe Embryonalentwicklung zu beurteilen. Heute ist es weniger die
Frage, ob Nahrungsergänzungen die Fruchtbarkeit verbessern können, sondern welche und in welcher Kombination.

\section{Hilfreiche Nahrungsergänzungen für den Mann}

Hilfreiche Nahrungsergänzungen, die in Studien eine Verbesserung des Spermiogramms erreichten, wurden gerade zusammenfassend publiziert [11], daher gehe ich hier nicht auf Einzelheiten ein. Zu diesen gehören (Dosierungen und Kombinationen variabel): Zink, Selen, Folsäure, Vitamin E (auch in Kombination mit Clomifen), Coenzym Q10, DHA, L-Carnitin, L-Arginin, Acetylcystein, Myo-Inositol. Besonders wirksam ist Myo-Inositol offenbar bei Männern mit Metabolischem Syndrom. Obwohl Spermiogramme bei Männern mit guter Vitamin-D-Versorgung besser waren als bei schlechter Vitamin-D-Versorgung, sind Supplementierungsstudien nicht eindeutig [1][2]. Sinnvoll ist es in jedem Fall, im Serum/Vollblut die Spiegel der wichtigsten Vitalstoffe zu überprüfen und dann gezielt Mängel auszugleichen. Die Supplementierung sollte mindestens über 3, besser über 6 Monate mit Spermiogrammkontrolle erfolgen. Das für Frauen empfohlene Nahrungsergänzungsmittel PREGNASana ${ }^{\circledR}$ kann auch bei Männern in Kombination mit einer Darmsanierung zu unerwarteten Verbesserungen des Spermiogramms und Konzeptionen führen. Selbst bei einem schweren Oligo-Astheno-Teratozoospermie-Syndrom lohnt sich der Versuch der Verbesserung von Lifestylefaktoren und der Gabe von ausgewogenen Nahrungsergänzungen [17].

\section{Hilfreiche Nahrungsergänzungen für die Frau}

Wurde eine hormonelle Kontrazeption beendet, dann ist die Nahrungsergänzung mit verschiedenen Vitalstoffen ein Muss, denn Hormone sind Vitaminräuber: Sämtliche B-Vitamine, Magnesium, Zink, Vitamin C und Jod werden offenbar vermehrt verbraucht oder ausgeschieden. Obwohl bekannt ist, dass Frauen mit einer guten Versorgung an Vitalstoffen bessere Fertilitätschancen haben als Frauen mit Defiziten, wird der Status nicht regelmäßig durch geeignete Blutuntersuchungen überprüft. In einem großen italienischen Kinderwunschzentrum wurden solche Überprüfungen kürzlich vorgenommen: von 269 Frauen (Durchschnittsalter 37 Jahre) hatten nur 69\% normale Homocysteinwerte und $44 \%$ normale $\mathrm{B}_{12}$-Konzentrationen. Nur $12 \%$ wiesen Erythrozytenfolatwerte, die als optimal für die Verhinderung eines Neuralrohrdefekts gelten, auf [15].

Im vergangenen Jahr erschien eine systematische Literaturübersicht zur orthomolekularen Therapie im Zusammenhang mit IVF [4]. Fünf Studien mit insgesamt 467 Teilnehmerinnen erfüllten die Kriterien. Obwohl auch hier die Therapien sehr heterogen waren, waren Schwangerschafts- und Lebendgeburtraten in den Orthomolekulariagruppen überzeugend besser als in den Kontrollgruppen. Eine italienische Arbeitsgruppe verglich bei über 39-jährigen Frauen jeweils 2 IVF-Zyklen, einen ohne und einen nach 3-monatiger Therapie mit Antioxidantien. Nach der 
Antioxidantientherapie war die Qualität der Oozyten signifikant besser und die Follikelflüssigkeit bot optimalen Schutz [16].

Eine besondere Herausforderung stellen Patientinnen mit PCO dar, da das Risiko der Überstimulation sehr hoch ist und die Qualität der Oozyten oft zu wünschen übriglässt. Bei Frauen mit niedrigen Vitamin-D-Spiegeln verbesserten sich zahlreiche Stoffwechsel- und Fertilitätsparameter durch eine 2-monatige Aufdosierung mit 50000 IE Vitamin D pro Woche [14]. Ein optimaler Vitamin-D-Spiegel erwies sich in einer anderen Studie als guter prognostischer Parameter, wobei die Rate von Lebendgeburten am höchsten war, wenn $25 \mathrm{OH}$-Vitamin-D über $45 \mathrm{ng} / \mathrm{ml}$ betrug [22]. Es gibt Hinweise dafür, dass durch hohe Vitamin-D-Konzentrationen in der Follikelflüssigkeit qualitativ hochwertigere Spermien selektioniert werden [6].

Ein relativ neuer Aspekt wurde bei PCO-Patientinnen bestätigt: die Gabe von Myo-Inositol verbessert nicht nur den Zuckerstoffwechsel, sondern auch die Fertilität. In einer randomisierten Studie konnte gezeigt werden, dass Frauen unter Myo-Inositol weniger follikelstimulierendes Hormon (FSH) bei der Follikelstimulation benötigten und eine bessere Schwangerschaftsrate erzielt werden konnte [8]. In einer anderen Studie wurden Myo-Inositol und Melatonin vom ersten Zyklustag bis 14 Tage nach dem Embryotransfer verabreicht: Oozyten- und Embryoqualität verbesserten sich signifikant [21]. Empfohlen wird eine Dosis von 2 g Myo-Inositol, 2 × täglich [9]. Auch für Frauen gilt, dass durch eine Laboruntersuchung der Versorgungstatus dokumentiert werden sollte. Optimale Vitamin-D-Werte sollten dauerhaft über $45 \mathrm{ng} / \mathrm{ml}$ Serum liegen.

Die kanadische Ärzteschaft hält es für sinnvoll, dass jede Frau in der generativen Phase ein Multivitamin- und Mineralprodukt mit $0,4 \mathrm{mg}$ Folat einnehmen sollte, da zu viele Schwangerschaften unverhofft eintreten würden und dann das Risiko für Missbildungen erhöht sei [26]. Bei anamnestisch erhöhtem Missbildungsrisiko sollte dann ein zusätzliches Folatpräparat verordnet werden. Auch in Deutschland werden solche Multivitamintabletten beworben. Wichtig ist, Produkte ohne Konservierungs- und Farbstoffe, ohne weitere E-Stoffe und ohne künstliche Süßstoffe zu bevorzugen. Andernfalls kann die Einnahme von Nahrungsergänzungen eher schaden als nutzen. Nach meiner Erfahrung eignet sich PREGNASana ${ }^{\circledR}$, da es keine Zusatzstoffe enthält.

\section{Probiotika zur Steigerung der Fertilität}

Der vaginale $\mathrm{pH}$-Wert ist bei Frauen niedriger als bei allen anderen Säugetieren. Im Vergleich zu anderen Säugetieren (max. $1 \%$ ) besteht die Vaginalflora zu über $70 \%$ aus Lactobazillen [19]. Die $\mathrm{H}_{2} \mathrm{O}_{2}$-bildenden Milchsäurebakterien haben die Eigenschaft, das Vaginalepithel zu schützen und pathogene Keime anzugreifen [27]. Bei einem optimalen Scheidenmilieu sind die Chancen für eine spontane
Konzeption besser als bei einer Fehlbesiedelung der Scheide. Empfehlenswert sind milchsäurehaltige Probiotika, die per oral verabreicht werden oder direkt über die Scheide.

\section{Pflanzenheilmittel}

Es gibt zwar typische Pflanzenheilmittel bei Fertilitätsstörungen, aber nur wenige Studien, auf die sich ein Therapeut beziehen kann. Agnus castus, der Mönchspfeffer, ist wohl die Pflanze, für die die beste Evidenz vorliegt. In einem Review über 13 randomisierte Studien kann der Einsatz von Agnus castus beim Prämenstruellen Syndrom (PMS), bei prämenstrueller Dysphorie und latenter Hyperprolaktinämie als berechtigt angesehen werden [7]. Es sollten standardisierte Extrakte gewählt (Agnolyt ${ }^{\circledR}$, Agnucaston ${ }^{\circledR}$, Femicur ${ }^{\circledR} N$ ) und über mindestens drei Monate während des gesamten Zyklus therapiert werden.

In dem Buch „Das alternative Kinderwunschbuch“ von Margret Madejsky gibt es eine Fülle von Anleitungen, um Frauen und Männer mit den unterschiedlichsten Fertilitätsstörungen mit Naturheilmitteln zu begleiten. Von der Entgiftung über die Optimierung der Ovarial- und Schilddrüsenfunktion bis hin zur postoperativen Nachsorge können Pflanzen der Traditionellen Europäischen Medizin (TEM) sinn- und erfolgreich eingesetzt werden.

\section{Homöopathie}

In der Ambulanz für Naturheilkunde wurden Pilotstudien bei infertilen Paaren mit Homöopathie durchgeführt. Bei infertilen Männern mit Oligoasthenozoospermie konnten signifikante Verbesserungen des Spermiogramms mit Einzelmitteln erzielt werden, sofern keine Umweltbelastungen oder chronischen Entzündungen vorlagen [10].

Frauen wurden in Abhängigkeit von den nachgewiesenen Sterilitätsursachen in verschiedenen Studien mit Komplex- oder Einzelmitteln behandelt. Komplexmittel mit Vitex agnus castus verbesserten die Lutealinsuffizienz, mit Einzelmitteln konnten Schwangerschaften erzielt werden. Der Therapieaufwand ist groß, sodass in Einzelfällen homöopathische Behandlungen sicher sinnvoll sind, für das Gros der infertilen Paare aber eher nicht in Frage kommen.

\section{Traditionelle Chinesische Medizin (TCM)}

Durch die zunehmende Akzeptanz der Akupunktur im Westen wird diese hierzulande auch bei Fertilitätsstörungen eingesetzt. Es zeigte sich, dass die Ergebnisse verbessert werden können, wenn nicht nur Nadeln benutzt werden, sondern auch die chinesische Kräutermedizin bzw. weitere Säulen der TCM berücksichtigt werden. In einer Metaanalyse, in der 40 randomisierte Studien mit über 4000 unfruchtbaren Frauen ausgewertet wurden, verdoppelte sich die Schwangerschaftsrate innerhalb von 3-6 Monaten, wenn chinesische Kräuter eingesetzt wurden, im Vergleich zur klassischen westlichen Medizin [23]. Eingeschlossen waren Frauen mit Endometriose, PCO, schlech- 
ter Tubendurchgängigkeit, Anovulation und idiopathischer Infertilität.

In einer retrospektiven Kohortenstudie aus den USA wurden 1000 IVF-Zyklen untersucht, wobei eine Gruppe nur IVF erhielt, eine Gruppe Akupunktur beim Embryotransfer und eine dritte TCM mit allen Aspekten [13]. Die Schwangerschaftsrate war nach TCM doppelt bis dreimal so hoch wie in der konventionellen Gruppe. In Deutschland behandelte Dr. Annemarie Schweizer-Arau Patientinnen mit schwerer Endometriose mit TCM und Hypnotherapie (SART). Damit erzielte sie eine signifikante und dauerhafte Schmerzreduktion und eine Schwangerschaftsrate von 55 \% [18]. Auch bei PCO können chinesische Kräuter punkten. Für eine Metaanalyse wurden 33 Studien ausgewählt, in denen sechs verschiedene Kräuter eingesetzt worden waren, die unterschiedliche Effekte auslösten [3]: LH-Suppression, Zyklusverbesserung, Besserung des Zuckerstoffwechsels und Eintritt von Schwangerschaften.

\section{Fazit}

In verschiedenen Studien konnte gezeigt werden, dass alleine die Berücksichtigung und Verbesserung der Lebensstilfaktoren die spontane Schwangerschaftsrate mindestens verdoppeln kann. Von wissenschaftlicher Seite sind die zuverlässigsten Daten zu Naturheilkunde und Kinderwunsch Studien, die sich auf die Optimierung des Lebensstils, der Ernährung und Nahrungsergänzung beziehen. Es ist jedoch keine Frage, dass die europäische und chinesische Pflanzenheilkunde noch viele Schätze bergen, mit denen der geschulte Therapeut infertile Paare unterstützen kann. Nach einer Basisdiagnostik der möglichen Sterilitätsursachen kann vielen Paaren durchaus empfohlen werden, diese Methoden zu nutzen, ehe sie sich auf die Methoden der assistierten Reproduktionsmedizin einlassen.

Interessenkonflikt

Die Autorin erklärt, dass keine wirtschaftlichen oder persönlichen Verbindungen bestehen.

Literatur

[1] Abbasihormozi S et al. Association of vitamin D status with semen quality and reproductive hormones in Iranian subfertile men. Andrology 2017; 5(1): 113-118

[2] Akhavizadegan H, Karbakhsh M. Comparison of serum vitamin $\mathrm{D}$ between fertile and infertile men in a vitamin $\mathrm{D}$ deficient endemic area: a case-control study. Urologia 2017; 84(4): 218-220

[3] Arentz $S$ et al. Herbal medicine for the management of polycystic ovary syndrome (PCOS) and associated oligo/amenorrhoea and hyperandrogenism; a review of the laboratory evidence for effects with corroborative clinical findings. BMC Complement Altern Med 2014; 14: 511

[4] Arhin SK et al. Effect of micronutrient supplementation on IVF outcomes: a systematic review of the literature. Reprod Biomed Online 2017; 35(6): 715-722

[5] Barisic A et al. Matrix metalloproteinase and tissue inhibitors of metalloproteinases gene polymorphisms in disorders that influence fertility and pregnancy complications: A systematic review and meta-analysis. Gene 2018; 647: 48-60

[6] Bollehuus Hansen L et al. Selection of High-Quality Spermatozoa May Be Promoted by Activated Vitamin D in the Woman. J Clin Endocrinol Metab 2017; 102(3): 950-961

[7] Die van MD et al. Vitex agnus-castus extracts for female reproductive disorders: a systematic review of clinical trials. Planta Med 2013; 79(7): 562-75

[8] Emekci Ozay O et al. Myo-inositol administration positively effects ovulation induction and intrauterine insemination in patients with polycystic ovary syndrome: a prospective, controlled, randomized trial. Gynecol Endocrinol 2017; 33(7): 524-528

[9] Facchinetti F et al. Results from the International Consensus Conference on Myo-inositol and d-chiro-inositol in Obstetrics and Gynecology: the link between metabolic syndrome and PCOS. Eur J Obstet Gynecol Reprod Biol 2015; 195: 72-6

[10] Gerhard I, Wallis E. Individualized homeopathic therapy for male infertility. Homeopathy 2002; Jul 91(3): 133-144 
[11] Gerhard I. Schritt für Schritt zum Baby. Co.med 2018; 24(07): 10-13

[12] Hart R et al. Periodontal disease: a potential modifiable risk factor limiting conception. Hum Reprod 2012; 27(5): 1332-42

[13] Hullender Rubin LE et al. Impact of whole systems traditional Chinese medicine on in-vitro fertilization outcomes. Reprod Biomed Online 2015; 30(6): 602-12

[14] Irani $M$ et al. Vitamin D ecreases serum VEGF correlating with clinical improvement in vitamin D-deficient women with PCOS: a randomized placebo-controlled trial. Nutrients 2017; 9, 334, 1-8

[15] La Vecchia I et al. Folate, homocysteine and selected vitamins and minerals status in infertile women. Eur ] Contracept Reprod Health Care 2017; 22(1): 70-75

[16] Luddi A et al. Antioxidants reduce oxidative stress in follicular fluid of aged women undergoing IVF. Reprod Biol Endocrinol 2016; 14(1): 57

[17] Magdi $Y$ et al. Effect of modifiable lifestyle factors and antioxidant treatment on semen parameters of men with severe oligoasthenoteratozoospermia. Andrologia 2017; 49(7)

[18] Meissner K, Bohling B, Schweizer-Arau A. Long-term effects of traditional Chinese medicine and hypnotherapy in patients with severe endometriosis--a retrospective evaluation. Forsch Komplementmed 2010; 17(6): 314-20

[19] Miller EA et al. Lactobacilli dominance and vaginal pH: why is the human vaginal microbiome unique? Front Microbiol 2016; 7: 1936

[20] Mumford SL et al. Higher urinary lignan concentrations in women but not men are positively associated with shorter time to pregnancy. J Nutr 2014; 144(3): 352-8

[21] Pacchiarotti A et al. Effect of myo-inositol and melatonin versus myo-inositol, in a randomized controlled trial, for improving in vitro fertilization of patients with polycystic ovarian syndrome. Gynecol Endocrinol 2016; 32(1): 69-73

[22] Pal L et al. Vitamin D status relates to reproductive outcome in women with polycystic ovary syndrome: secondary analysis of a multicenter randomized controlled trial. J Clin Endocrinol Metab 2016; 101(8): 3027-35

[23] Ried K. Chinese herbal medicine for female infertility: an updated meta-analysis. Complement Ther Med 2015; 23(1): $116-28$

[24] Vanegas JC et al. Soy food intake and treatment outcomes of women undergoing assisted reproductive technology. Fertil Steril 2015; 103(3): 749-55.e2

[25] Volkmann P. Parodontitis und Organstörungen. Angew Komplementärmed 2018; 09: 2-11

[26] Wilson RD et al. Pre-conception folic acid and multivitamin supplementation for the primary and secondary prevention of neural tube defects and other folic acid-sensitive congenital anomalies. J Obstet Gynaecol Can 2015; 37(6): 534-52

[27] Witkin SS, Linhares IM. Why do lactobacilli dominate the human vaginal microbiota? Bjog 2017; 124(4): 606-611

\section{Weiterführende Literatur}

Bihlmaier S. Tomatenrot und Drachengrün. Weil der Stadt: Hädecke; 2018

Gerhard I. Myome selbst heilen. Richtig ernähren - die natürliche Alternative zu Pillen und OPs. Murnau: Mankau; 2018

Hühn S. Raum für deine innere Frau. Darmstadt: Schirner; 2018
Jacob L. Dr. Jacobs Weg des genussvollen Verzichts: Die effektivsten Maßnahmen zur Prävention und Therapie von Zivilisationskrankheiten. Heidesheim a. R.: nutricaMEDia; 2013

Madejsky M. Das alternative Kinderwunschbuch. München: arkana; 2015

Ohlig A. Die bewegte Frau. Luna Yoga für Gesundheit und Lebenslust. München: Nymphenburger; 2016

Pfeiffer C. Therapeutische Frauen-Massage. Saarbrücken: Edition Lehrwerk; 2016

Pröll G. Die „glückliche“ Gebärmutter: Innere Bilder - selbstheilende Kraft bei Unterbauchbeschwerden: Mit praktischen Übungen nach der Methode Wildwuchs. Würzburg: Diametric; 2016

Schweizer Arau A. Der sanfte Weg zum Wunschkind. München: Kösel; 2015

Volkmann P-H. Darm gesund - Mensch gesund! Ganz einfach! Wieder fit durch gesunde Ernährung. 1st ed. Lübeck: VBN; 2017

Wischmann T, Stammer H. Der Traum vom eigenen Kind: Psychologische Hilfen bei unerfülltem Kinderwunsch (Rat \& Hilfe). Stuttgart: Kohlhammer; 2016

Zart B. Kinderwunsch, die besten Rezepte, um natürlich schwanger zu werden. München: Gräfe und Unzer; 2017

\section{Autorin}

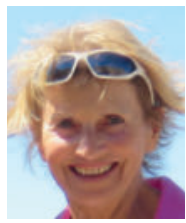

\section{Ingrid Gerhard}

Ingrid Gerhard ist Fachärztin für Frauenheilkunde, Naturheilverfahren, Umweltmedizin. Bis 2002 praktizierte, forschte und lehrte sie an der Abteilung für Gynäkologische Endokrinologie und Fertilitätsstörungen der Univ. Frauenklinik in Heidelberg. Dort baute sie in den 1980er-Jahren die Ambulanz für Naturheilkunde auf. Seit 2002 ist sie als freie Dozentin und Autorin tätig. Sie publizierte neben Lehrbüchern auch Bücher und Ratgeber für Laien. Seit 10 Jahren betreibt sie das Webmagazin www.netzwerk-frauengesundheit.com.

\section{Korrespondenzadresse}

\section{Prof. Dr. med. Ingrid Gerhard}

Fachärztin für Frauenheilkunde, Naturheilverfahren, Umweltmedizin

Albert-Überle-Str. 11

69120 Heidelberg

E-Mail: ingerhard@gmail.com

Internet: www.netzwerk-frauengesundheit.com

\section{Bibliografie}

DOI https://doi.org/10.1055/a-0760-9062

EHK 2018; 67: 329-336

(c) MVS Medizinverlage Stuttgart GmbH \& Co. KG ISSN 0014-0082 